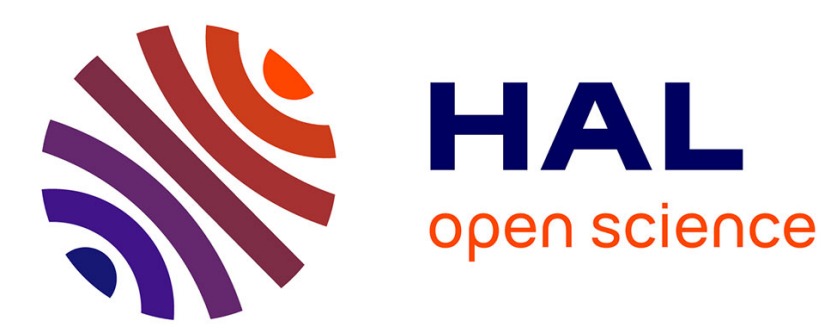

\title{
Investigation of the structures of sulfur on Mo(100) by scanning tunneling microscopy
}

J. Dunphy, P. Sautet, D.F. Ogletree, M.B. Salmeron

\section{To cite this version:}

J. Dunphy, P. Sautet, D.F. Ogletree, M.B. Salmeron. Investigation of the structures of sulfur on Mo(100) by scanning tunneling microscopy. Journal of Vacuum Science \& Technology A, 1993, A11, pp.1975-1982. 10.1116/1.578533 . hal-00006088

\section{HAL Id: hal-00006088 \\ https://hal.science/hal-00006088}

Submitted on 17 May 2020

HAL is a multi-disciplinary open access archive for the deposit and dissemination of scientific research documents, whether they are published or not. The documents may come from teaching and research institutions in France or abroad, or from public or private research centers.
L'archive ouverte pluridisciplinaire HAL, est destinée au dépôt et à la diffusion de documents scientifiques de niveau recherche, publiés ou non, émanant des établissements d'enseignement et de recherche français ou étrangers, des laboratoires publics ou privés.

\section{(c)(1)}

Distributed under a Creative Commons Attribution| 4.0 International License 


\title{
Investigation of the structures of sulfur on Mo(100) by scanning tunneling microscopy
}

\author{
J. C. Dunphy \\ Center for Advanced Materials, Materials Science Division, Lawrence Berkeley Laboratory, Berkeley. \\ California 94720 and Department of Physics, University of California at Berkeley, Berkeley, \\ California 94720 \\ P. Sautet, a) D. F. Ogletree, and M. B. Salmeron \\ Center for Advanced Materials, Materials Science Division, Lawrence Berkeley Laboratory, Berkeley, \\ California 94720
}

The structures of the four ordered overlayers of sulfur on the Mo(100) surface were investigated by scanning tunneling microscopy (STM). In order of increasing sulfur coverage, the overlayers have $c(2 \times 2),\left\{\begin{array}{ll}2 & 1 \\ 1 & 1\end{array} \mid, c(4 \times 2)\right.$, and $p(2 \times 1)$ low energy electron diffraction (LEED) patterns. Only the $c(2 \times 2)$ overlayer geometry has been determined from LEED $I(V)$ analysis. An examination of point and line defects and domain boundaries in STM images provides information beyond that known from LEED on the required symmetries of the ordered overlayers. Several proposed structures were shown to be incompatible with these symmetries and therefore discarded. Only one model of the $c(4 \times 2)$ structure was found to be consistent with the symmetry of the STM images. This structure contains sulfur atoms occupying exclusively fourfold hollow sites. Unexpectedly, STM images of the surface with the $p(2 \times 1)$ LEED pattern did not have $p(2 \times 1)$ symmetry, but consisted of small domains of the $c(4 \times 2)$ structure.

\section{INTRODUCTION}

Molybdenum single crystals surfaces are of interest as a model for industrial molybdenum sulfide catalysts. ${ }^{1,2}$ A number of studies ${ }^{1-10}$ have been published on the sulfided Mo(100) surface. These studies have determined that sulfur forms several ordered structures as a function of coverage. While early reports ${ }^{3-6}$ confficted over the order and number of distinct low energy electron diffraction (LEED) patterns, more recent results ${ }^{7-10}$ agree on four LEED patterns and the sulfur coverages at which they appear. However, except for the $c(2 \times 2)$ ordered overlayer, the structures of the unit cells which produce these LEED patterns are unknown. Several models have been proposed ${ }^{3-5}$ for each of the other overlayers. Scanning tunneling microscopy (STM), by providing information complimentary to the LEED results, should be helpful in determining which of the proposed models is correct.

The most recently published studies ${ }^{7-10}$ of $\mathrm{S}$ on $\mathrm{Mo}(100)$ agree on four ordered structures. At coverages of approximately $0.5,0.67,0.75$, and 1 monolay of sulfur the reported LEED patterns have $c(2 \times 2),\left.\right|_{1} ^{2}|1|, c(4 \times 2)$, and $p(2 \times 1)$ symmetry. These coverages correspond to one, two, three, and two atoms per unit cell, respectively. A LEED calculation performed on the $c(2 \times 2)$ structure determined that the sulfur atoms reside at fourfold hollow sites. ${ }^{5}$ A later dynamical LEED ${ }^{11}$ and a tensor LEED ${ }^{12}$ calculation determined the precise position of the sulfur adatoms and the displacements of the substrate Mo surface

a) Permanent address: Laboratoire de Chimie Théorique, ENS Lyon 46, allée d'Italie, 69464 Lyon Cedex 07. France and Institut de Recherche sur la Catalyse 2, Avenue A. Einstein, 69626 Villeurbanne Cedex, France. atoms in this structure. Dynamical LEED calculations have also been attempted for the $p(2 \times 1)$ overlayer, but an adequate fit between the calculation and experimental $I(V)$ curves has not been obtained. ${ }^{13}$ A previous STM study ${ }^{14}$ of this structure in air favored a model with all sulfur atoms occupying an asymmetric site between hollow and bridge. However, the symmetries of this structure would cause the extinction of some spots in the LEED pattern, which is not observed experimentally. A total energy calculation favored a model with half the sulfur atoms in hollow sites and the other half in bridge sites. ${ }^{15}$

In our attempt to determine the structure of these overlayers we considered models with sulfur atoms in bridge and hollow sites. Unfortunately, we were not able to directly determine which of these structures is correct. Although the unit cell periodicity is resolved by the STM, the constituent atoms are not resolved individually except in the simple $c(2 \times 2)$ structure. However, proposed structures could be eliminated from consideration by showing them to be incompatible with observations of the symmetry of the overlayer and of point defects and domain boundaries.

\section{EXPERIMENT}

Sample preparation and STM imaging were performed inside a standard surface science ultra high vacuum (UHV) chamber with a base pressure of $5 \times 10^{-10}$ Torr. Resides the STM, the chamber was equipped with Auger electron spectroscopy (AES), LEED, and $\mathrm{Ar}^{+{ }^{+}}$sputtering. The design of the STM is described elsewhere. ${ }^{16}$ Mechanically cut $\mathrm{Pt} / \mathrm{Rh}(80 \% / 20 \%)$ tips were used. The single crystal sample could be separately heated with an electron 
beam heater and cooled by placing the sample holder in contact with a copper block cooled by liquid nitrogen. STM imaging took place at room temperature.

The single crystal sample was approximately $1 \mathrm{~cm}$ diam and $<1 \mathrm{~mm}$ thick. It was oriented to within $<1.5^{\circ}$ of the [100] direction and polished using standard metallurgical techniques. Carbon and other impurities were removed by sputtering while heating to approximately $600 \mathrm{~K}$. The carbon remaining after this treatment was removed by repeated heating in oxygen $\left(5 \times 10^{-7}\right.$ Torr at $\left.1600 \mathrm{~K}\right)$. Remaining oxygen was removed by heating the crystals in UHV above $1900 \mathrm{~K}$. Careful cleaning of the crystal was important to insure that only sulfur structures were imaged with the STM. Carbon and oxygen, the most common impurities on the surface, form ordered structures with some of the same LEED patterns as sulfur. Unless the bulk is depleted of these impurities to a sufficient degree they will segregate back to the surface while the crystal is cooled.

Sulfur was deposited on the clean surface by decomposition of $\mathrm{H}_{2} \mathrm{~S}$ gas and with a solid-state electrochemical source $\left(\mathrm{Ag} / \mathrm{AgI} / \mathrm{Ag}_{2} \mathrm{~S}\right) \cdot{ }^{17}$ While the lower coverage structures could be produced easily by $\mathrm{H}_{2} \mathrm{~S}$ exposure, the electrochemical source was necessary to cover the surface with enough sulfur to produce the highest coverage $p(2 \times 1)$ overlayer. The crystal was heated during exposure to $\mathrm{H}_{2} \mathrm{~S}$ gas, while it was kept at room temperature during exposure to sulfur from the electrochemical source.

Prior to imaging of the sulfur overlayer, AES was used to determine sulfur coverage on the surface. The sample was annealed to produce an ordered LEED pattern. STM images of the surface were obtained using several different tips and preparations of each structure. Several hundred STM images of the overlayer structures were obtained. As discussed in a separate paper, ${ }^{10}$ sulfur adsorption was found to modify the structure of the atomic steps on the surface by causing the coalescence of steps and the consequent enlargements of the (100) terraces.

The surface was imaged in both constant current (topographic) and constant height mode. In most of the images the sample was biased negative relative to the tip. Changing the polarity of the bias was found to have no effect on the images. The tunneling current was set at between 1 and $3 \mathrm{nA}$ and the bias voltage between 10 and 100 $\mathrm{mV}$. Decreasing the gap resistance tended to increase the corrugation of the sulfur structures. Changing the bias had little effect on the image as long as the gap resistance was kept constant.

Thermally induced drifts of the sample during imaging significantly distorted the images, particularly the topographic mode images that require longer acquisition time. The correct shape and size of the images was determined from current mode images acquired quickly enough so that the drift had little effect on them. Using this information the images were replotted with the correct shape using a two-dimensional second-order fit of the lattice. All the images shown in this paper are drift-corrected topographic mode images. To make interpretation easier, the images are rotated so that the horizontal and vertical directions correspond to the [010] and [001] directions of the Mo sub-

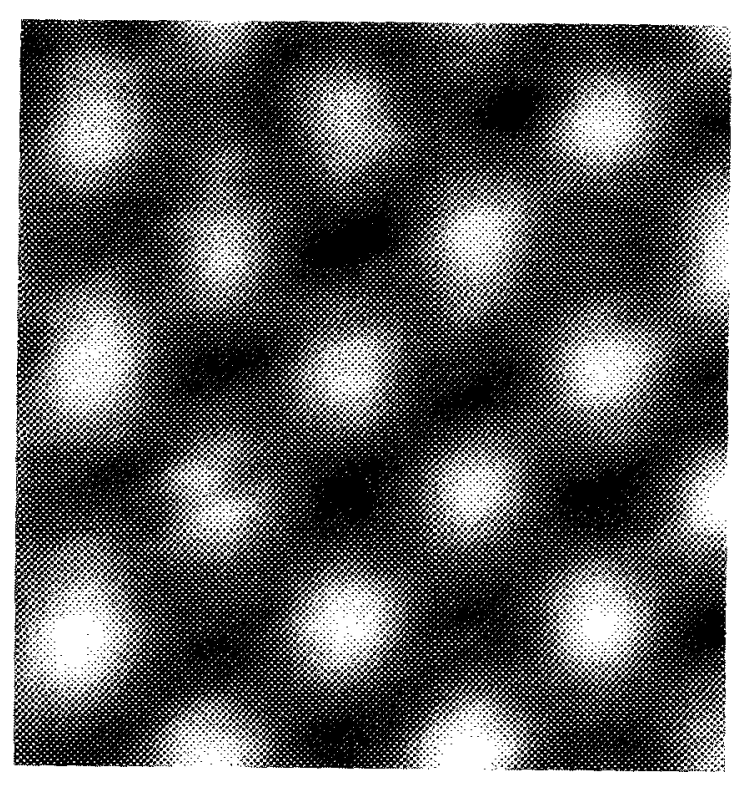

FIG. 1. An $18 \times 19 \AA$ A topographic STM image of the $c(2 \times 2)$ structure of $\mathrm{S}$ on $\mathrm{Mo}(100)$. The lattice directions of the Mo substrate are the horizontal and vertical directions in the image. The bright maxima in the image are $0.22 \AA$ in height. Point defects appear as missing maxima in STM images indicating that these maxima correspond to the position of sulfur atoms on the surface. Bias $=15 \mathrm{mV}, I=1.0 \mathrm{nA}$.

strate. They were also Fourier filtered to remove spatial frequency components too high to represent real information.

\section{RESULTS}

\section{A. $c(2 \times 2)$ structure}

Images of the $\mathrm{Mo}(100)$ surface with half a monolayer of sulfur and a $c(2 \times 2)$ LEED pattern show ordered regions approximately $20-50 \AA$ across separated by partially ordered regions. The boundaries between these regions run in the [010] directions of the substrate. The partially ordered regions contained maxima with approximately $0.8 \AA$ corrugation which were commonly, but not always, spaced by twice the Mo lattice constant $a$ of $3.15 \AA$. As the AES spectrum showed little carbon or oxygen contamination and the disordered regions were common on the surface, it is likely that these areas are due to a different structure of sulfur and not to an impurity. They probably consist of sulfur which has not ordered into the $c(2 \times 2)$ structure due to insufficient annealing or too low a sulfur coverage. These regions may produce the $p(2 \times 2)$ and $c(4 \times 4)$ LEED patterns which have been reported by some authors ${ }^{5-7}$ at coverages well under half a monolayer.

An example of an image of an ordered region of this surface is shown in Fig. 1. The structure in the image is consistent with the observed LEED pattern. Rows of nearest-neighbor maxima run at $45^{\circ}$ to the lattice directions of the substrate. Also the measured spacing between the maxima is within $10 \%$ of the $4.7 \AA$ nearest-neighbor distance of a $c(2 \times 2)$ overlayer. The corrugation of the maxima in this image is $0.22 \AA$. Occasionally images of the $c(2 \times 2)$ structure were obtained which contained a smaller 


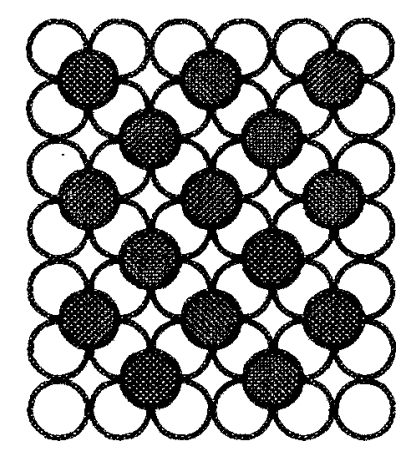

FiG. 2. A model of the hollow site $c(2 \times 2)$-S overlayer.

secondary maximum at the center of four primary maxima. We believe that these images were produced by a different tip end structure and/or composition. Point defects in the images always appeared as a missing maximum. This demonstrates that the primary maxima in the image corresponds to the location of the sulfur atoms, in agreement with the positive corrugation observed for sulfur on other metal surfaces. ${ }^{18-21}$

As described previously, several LEED studies ${ }^{5,11,12}$ of this surface placed the sulfur atom in each unit cell at a fourfold hollow site as shown in Fig. 2. The STM images are consistent with this result. The maxima in the images have fourfold symmetry, which is inconsistent with any sulfur bonding sites except the hollow and top. Additionally, if the sulfur atoms reside at any site other than the hollow or the top site there would be at least two domains of this structure inequivalent except by a rotation by $90^{\circ}$. Sulfur atoms in these two domains should be separated across the boundary by half-integer lattice vectors as shown in Fig. 3. While many domain boundaries were observed in images of the $c(2 \times 2)$ structure, the maxima in the image were always found to be separated by an integer number of lattice vectors across these boundaries. While the top site is consistent with the symmetry of the images, sulfur has never been found to bind at these sites on any metal surfaces, ${ }^{22}$ so we consider this site unlikeily.

\section{B. $\left|{ }_{1}^{2} \bar{i}\right|$ structure}

It was difficult to obtain the proper sulfur coverage to produce a surface with a LEED pattern with only the spots

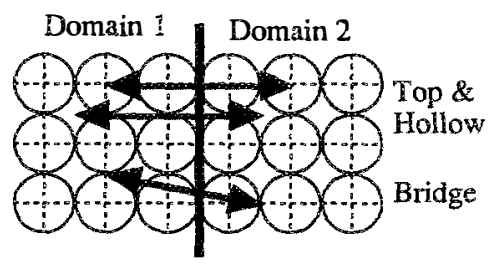

Fig. 3. A model of the Mo (100) surface showing the separation between high symmetry sites in two domains rotated by $90^{\circ}$ relative to each other. A $(1 \times 1)$ latice aligned on the fourfold symmetric top or hollow sites remains on the same points after a rotation through $90^{\circ}$. Conversely, a lattice aligned on bridge sites is displaced by a lattice vector of $[0, \pm a / 2$, $\pm a / 2$ ] after a $90^{\circ}$ rotation.

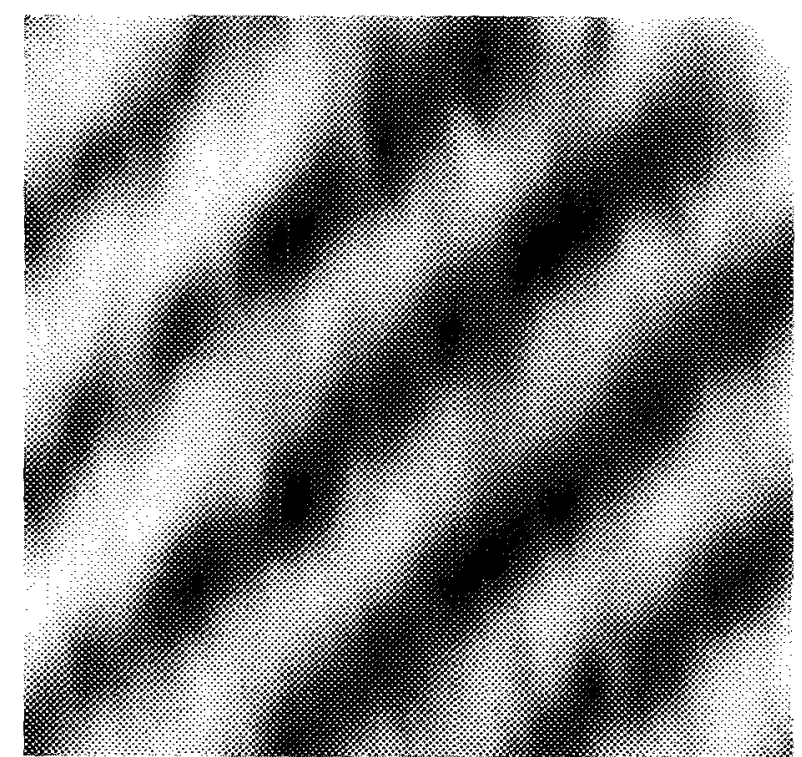

FIG. 4. A 25-Å square STM topographic image of the $\left|{ }_{i 1}^{2 i}\right|$ structure of $S$ on Mo(100). It consists of unresolved rows of sulfur atoms running diagonally in the image along the [011] direction. The lattice directions of the Mo substrate are the horizontal and vertical directions in the image. The corrugation of the rows is $0.27 \AA$. Bias $=100 \mathrm{mV}, I=1.0 \mathrm{nA}$.

of the $\left.\right|_{1} ^{2}{ }_{1}{ }_{1} \mid$ structure and no spots from either the $c(2 \times 2)$ or $c(4 \times 2)$ structures. In STM images the surface was always covered with domains of the $\left|\begin{array}{ll}2 & 1 \\ 1 & 1\end{array}\right|$ structure intermixed with areas of $c(2 \times 2)$ or $c(4 \times 2)$ ordered stilfur. An image of an area with the $\left.\right|_{1} ^{2}{ }_{1}$ structure is shown in Fig. 4. The rows in this image run in the [011] direction and are separated by three Mo lattice spacings as expected. The corrugation of the rows is $0.27 \AA$. The rows' appearance was dependent on the STM tip. While individual atoms within the rows were never resolved, the rows often contained half as many maxima as there are atoms in the overlayer. Occasionally the rows had a rippled appearance.

There are three possible high symmetry modeis for the $\left.\left.\right|_{2} ^{2} \begin{array}{l}1 \\ 1\end{array}\right]$ structure consisting of bridge and hollow sites. These are shown in Fig. 5. Unfortunately, the appearance of the
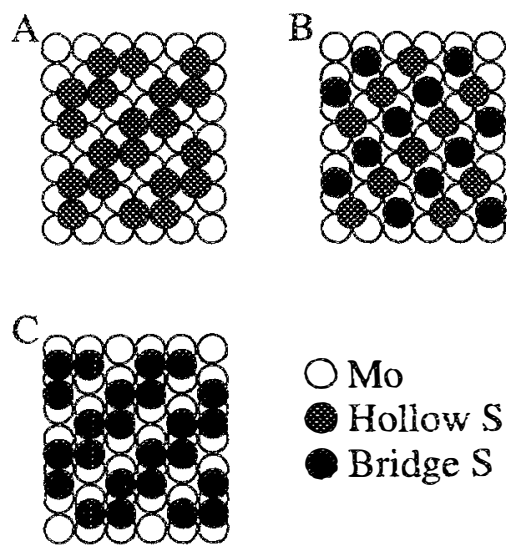

O Mo

Hollow $S$

Bridge $\mathrm{S}$

Fig. 5. Three models of the $\left.\right|_{1} ^{2}{ }_{1}^{1}$ structure with sulfur atoms at bridge and holiow sites. (A) all sulfur atoms at hollow site. (B) A mixture of hollow and bridge sites. (C) All sulfur atoms at bridge sites. 


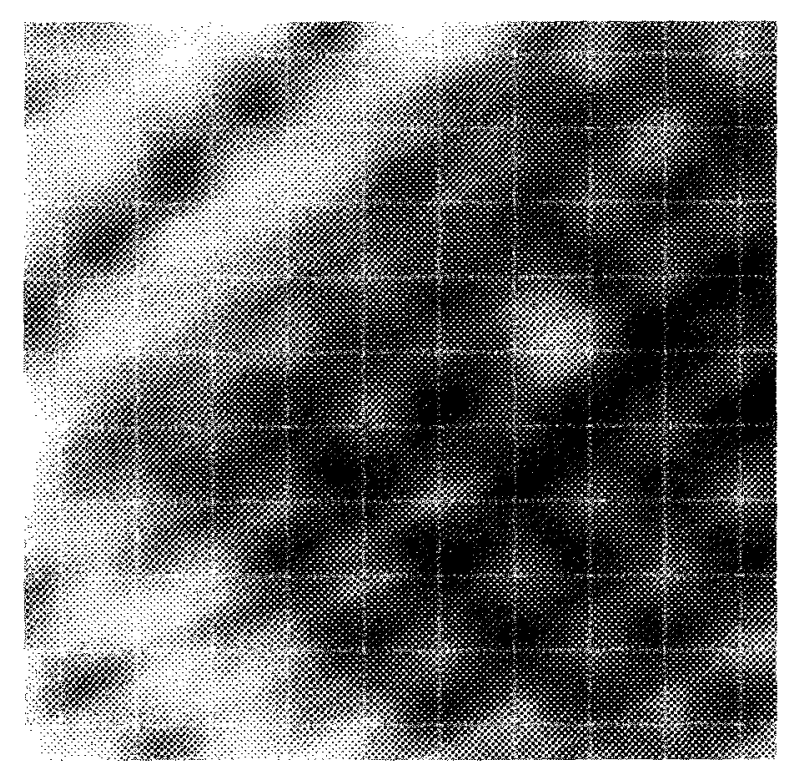

Fig. 6. A $31 \times 31 \AA$ topographic STM image showing the boundary region between the two domains of the $\left.\right|_{1} ^{2} \frac{1}{1} \mid$ structure (on the left, brighter rows) and the $c(2 \times 2)$ overiayer (on the right, dimmer spots) together. A $(1 \times 1)$ grid is drawn over the image and aligned to the $c(2 \times 2)$ overlayer. The cross marks on the grie are therefore positioned over fourfold hollow sites of the substrate. The grid shows that the rows of the $\left.\right|_{1} ^{2}{ }_{1}^{1} \mid$ structure appear to be centered over bridge sites. Bias $=50$ $\mathrm{mV} . I=1.0 \mathrm{nA}$.

STM images of this structure could be explained by any of the three models. If we assume that, as in the $c(2 \times 2)$ structure, the sulfur atoms have positive corrugation, the rows of sulfur atoms in Figs. 5(A) and 5(C) could correspond to the rows in the image. However, the rows of bridge sites in model $\mathrm{B}$ could also produce the rows in the image. As the bridge atoms are higher above the surface than atoms at hollow sites one would expect the rows of bridge site atoms in model B to appear brighter in an STM image than the rows of hollow site atoms. The observed variations in the experimental shape of the rows can be explained in all cases as the effect of a particular tip structure and are not helpful in determining the correct unit cell structure.

Since the $\left|\begin{array}{lll}2 & 1 \\ 1 & 1\end{array}\right|$ structure coexisted on the surface with the $c(2 \times 2)$ structure it was possible to determine the relative position of the rows and the maxima of the $c(2 \times 2)$ overlayer in the STM images. Figure 6 shows an image of the boundary region between both structures. In this image the $c(2 \times 2)$ domain occupies the right-hand side and its maxima have a low average intensity. Two domains of the $\left|\begin{array}{ll}2 & 1 \\ 1 & 1\end{array}\right|$ structure rotated by $90^{\circ}$ relative to each other appear as rows running diagonally up and down on the left-hand side. The intensity is higher in this region. A $(1 \times 1)$ grid is drawn over the image aligned to the maxima in the $c(2$ $\times 2)$ domain. If the hollow site $c(2 \times 2)$ model is correct, the top Mo substrate atoms are located at the center of the squares of the grid. The rows of the $\left.\right|_{1} ^{2}{ }_{1}^{1}$ ! structure appear to be centered over bridge sites of this lattice. This position is consistent with model B. If this is the correct model, the maxima at bridge sites appear elongated in one of the lat-

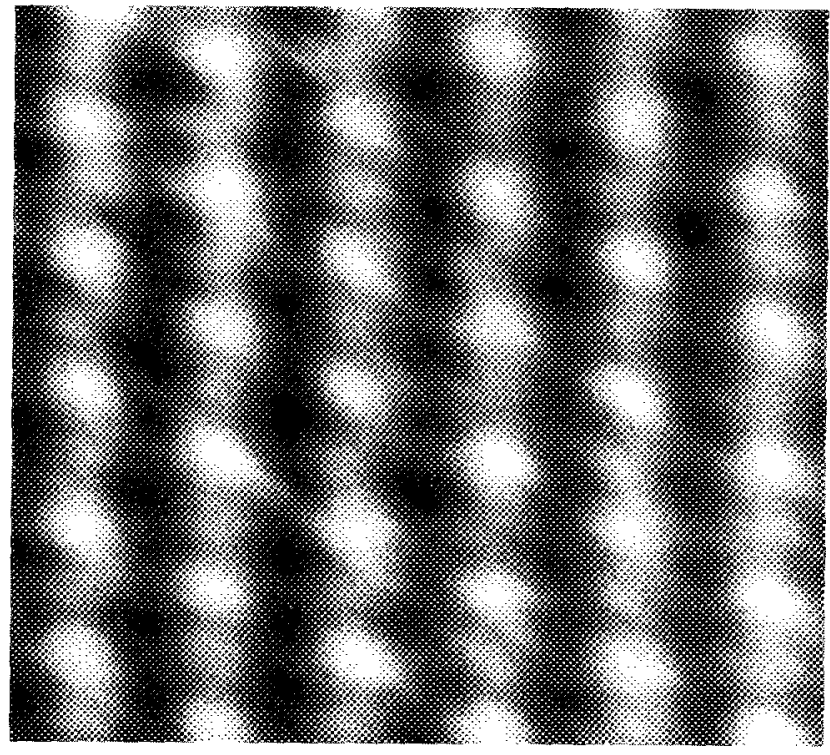

Fig. 7. A $38 \times 38 \AA$ STM topographic image of the $c(4 \times 2)$ structure. The long direction of the unit cell runs in the horizontal direction in this image. Each unit ceil contains one bright maxima which in joined by a dimmer region to other maxima along the short direction of the unit cell. There are two perpendicular mirror planes in each unit cell which cross at the maxima. The maximum corrugation between the rows is $0.45 \AA$. Bias $=50 \mathrm{mV}, I=2.8 \mathrm{nA}$.

tice directions. However, since we have not yet ascertained that sulfur atoms have positive corrugation in this structure, the result is not conclusive.

\section{C. $c(4 \times 2)$ structure}

At a coverage of approximately three quarters of a monolayer a sharp $c(4 \times 2)$ LEED pattern was obtained. STM images of the surface showed it was covered with ordered domains of approximately $100 \AA$ in size. An example of an image of a single domain is shown in Fig. 7. The staggered vertical rows of maxima in the figure are separated by twice the Mo lattice vectors. The maxima along the rows are also separated by this distance. The symmetry of the maxima in this image is $c(4 \times 2)$, consistent with the LEED pattern. There are two perpendicular mirror planes in each unit cell of the STM image which run through the maxima in the unit cells. Six possible model structures, shown in Fig. 8, have these two mirror planes and contain the three atoms per unit cell required to have 0.75 monolayers coverage. In models $\mathrm{A}-\mathrm{C}$ the perpendicular mirror planes cross at hollow sites while in models D-F they cross at bridge sites.

Half of these models can be discarded by observing the symmetry of domain boundaries in this structure. An image of the boundary between two domains of $c(4 \times 2)$ ordered sulfur rotated by $90^{\circ}$ relative to each other $[c(4 \times 2)$ and $c(2 \times 2)]$ is shown in Fig. 9. A $(1 \times 1)$ lattice is plotted over this image and lined up with the maxima of one domain. In the rotated domain the lattice is also found to line up with the maxima. This indicates that the maxima must be located at fourfold (hollow or top) sites on the lattice. 

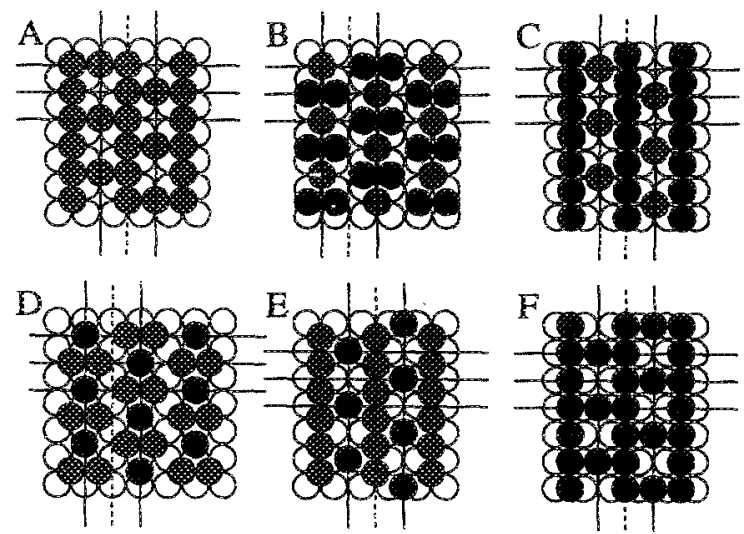

$\mathrm{Mo}$

Hollow $S$

Bridge $\mathrm{S}$

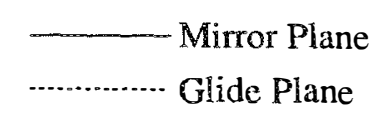

Fyg. 8. Models of the possible $c(4 \times 2)$ unit cells which contain three sulfur atoms and have the two perpendicular mirror planes (continuous lines) apparent in the STM images. The mirror planes in models A-C cross over hollow sites. In models D-F they cross over bridge sites. Glide planes are shown as dashed lines.

If they were located over twofold (bridge) sites one domain would be shifted by $[0, \pm a / 2, \pm a / 2]$ relative to the other domain as shown in Fig. 2. The lack of this shift was observed on all of the images of domain boundaries. The maxima are at points in the image where the two perpendicular mirror planes cross. Therefore, the mirror planes cross over hollow sites, and models $\mathrm{D}-\mathrm{F}$ must be discarded.

Observations of other domain boundaries support only one of the remaining models. Figure 10(a) shows an antiphase boundary in which one domain is shifted by one substrate lattice distance $a$ relative to the other. Figure $10(b)$ is an image of a domain boundary region between two perpendicular domains in which the domains appear to "mix" into a $(2 \times 2)$ structure. The structure in this area can be explained as a periodic array of antiphase boundaries like that shown in Fig. 10(a). This region appears to have a fourfold symmetry, and extends away from both the $c(4 \times 2)$ and $c(2 \times 4)$ domains symmetrically. As two do-

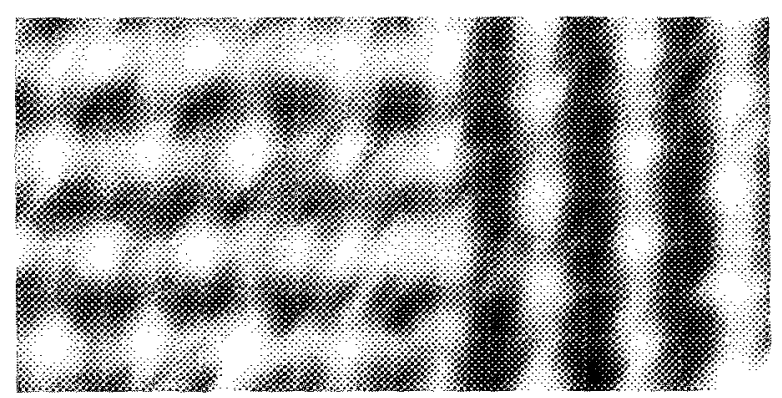

FIG. 9. A $50 \times 25 \AA$ STM topographic image showing two domains of the $c(4 \times 2)$ structure. The domain on the left is rotated $90^{\circ}$ relative to the domain on the right. A $(1 \times 1)$ grid is drawn over the image and aligned to the maxima of one domain. It is also found to be aligned with the maxima of the second domain. This indicates that the maxima in the image reside on fourfold symmetric sites. Bias $=100 \mathrm{mV}, z=1.0 \mathrm{nA}$.

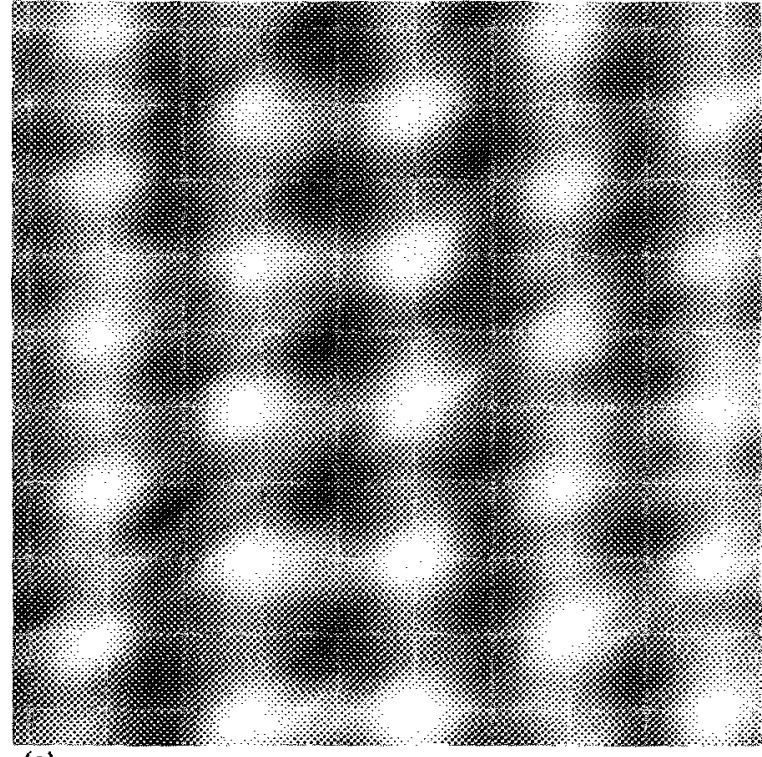

(a)

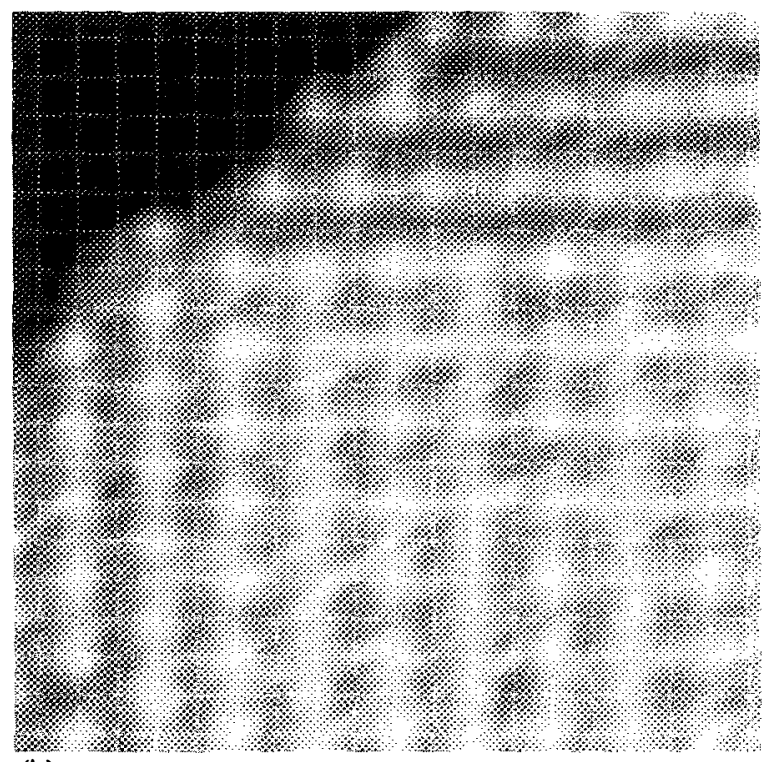

(b)

FIG. 10. Defects in the $c(4 \times 2)$ overlayer. (a) A $31 \times 31 \AA$ image of an antiphase boundary in which one domain is shifted by one Mo lattice spacing $a$ relative the other. Bias $=50 \mathrm{mV}, I=2.8 \mathrm{nA}$. (b) A $60 \times 6 \AA^{\circ}$ image of an area where two perpendicular domains of the $c(4 \times 2)$ overlayer appear to mix into a $(2 \times 2)$ structure. The fourfold symmetry of this structure is only compatible with the all hoilow site sulfur $c(4 \times 2)$ model. Bias $=100 \mathrm{mV}, I=1.0 \mathrm{nA}$.

mains of models B and C contain two different orientations of bridge sites, these models may not extend symmetrically into a $(2 \times 2)$ region. A periodic array of antiphase boundaries in the structure of model A would have fourfold symmetry. Therefore model $\mathbf{A}$, in which all sulfur atoms are located at hollow sites, is the most consistent with the STM images.

\section{D. " $p(2 \times 1)$ " structure}

Producing a sample with a sharp $p(2 \times 1)$ LEED pattern proved to be difficult. Since it is not possible to produce the required saturation coverage of sulfur by exposure 


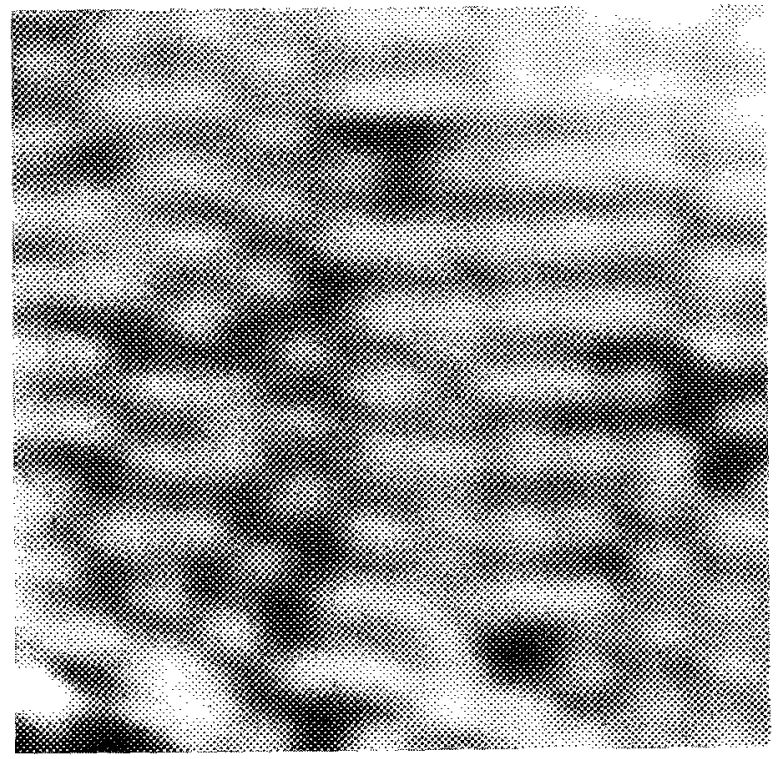

FIG. 11. A $70 \times 70 \AA$ image of the surface after a $\rho(2 \times 1)$ LEED patiern was obtained. The symmetry is not the expected $p(2 \times 1)$. The surface is instead covered by small domains of one to three unit cells with $c(4 \times 2)$ symmetry. Extra sulfur atoms at the domain boundaries increase the average sulfur coverage above that of the ordered $c(4 \times 2)$ overlayer. Bias $=100 \mathrm{mV}, I=1.0 \mathrm{nA}$.

to low pressures of $\mathrm{H}_{2} \mathrm{~S}$, the electrochemical sulfur source was used to deposit sulfur on the surface. Annealing of the surface was necessary to obtain an ordered LEED pattern. Excessive annealing produced the $c(4 \times 2)$ pattern while insufficient annealing left the LEED pattern diffuse. Previous reports ${ }^{4,5}$ of this structure have stated that the LEED pattern was often streaked, at least at some sulfur coverages. In the present experiment the overlayer spots of the LEED pattern were streaked in the direction of the quarter order spots of the $c(4 \times 2)$ LEED pattern at some electron energies. Adjusting the electron energy changed the intensity of the streaking; at some energies it was nearly invisible.

Unlike the previous lower coverage structures, we were unable to obtain STM images of a surface structure consistent with the symmetry of the LEED pattern. This result is different from the previous STM study of this surface in air in which images of a surface with apparent $p(2 \times 1)$ symmetry were obtained. ${ }^{14}$ Because of the air environment the observed $p(2 \times 1)$ ordering may be due to some impurity which was absent in our UHV experiment.

A representative image of the surface with a $p(2 \times 1)$ LEED pattern is shown in Fig. 11. It consists of very small domains of $c(4 \times 2)$ ordered sulfur. This image is not actually inconsistent with the LEED pattern, but only with its apparent $p(2 \times 1)$ symmetry. The $c(4 \times 2)$ LEED pattern contains all the spots of the $p(2 \times 1)$ pattern and additional quarter order spots. The streaking we observed in the $p(2 \times 1)$ LEED pattern was in the direction of these spots. The lack of large domains on the surface would cause streaking of the higher-order spots as they correspond to longer range order on the surface. The higher coverage of sulfur on this surface relative to the ordered $c(4 \times 2)$ overlayer may be explained by the large number of antiphase defects present which have a local coverage of one monolayer. As other authors have also reported streaking of the $p(2 \times 1)$ overlayer LEED spots, it is possible that all reports of the $p(2 \times 1)$ LEED pattern were obtained from surfaces with small $c(4 \times 2)$ domains. It may also be that some impurity is required to induce $p(2$ $\times 1$ ) ordering of the sulfur.

\section{CONCLUSION}

By careful analysis of the symmetry of STM images, particularly those containing point defects and domain boundaries, the range of possible structures of sulfur on Mo(100) was narrowed. STM images of the $c(2 \times 2)$ structure are consistent with the results obtained from dynamic LEED calculations. Of a number of proposed models of the $c(4 \times 2)$ structure only one model was found to be consistent with the STM images. This model consists of sulfur atoms adsorbed at fourfold hollow sites. Real space imaging by the STM has shown that the $p(2 \times 1)$ LEED pattern does not correspond to a true $p(2 \times 1)$ ordering. The structure of the $\left|\begin{array}{ll}2 & 1\end{array}\right|$ overlayer is still unknown.

\section{ACKNOWLEDGMENTS}

This work has been supported by the Director, Office of Energy Research, Office of Basic Energy Sciences, Materials Science Division of the U.S. Department of Energy under Contract No. DE-AC03-76SF00098. P.S. acknowledges the support of NATO.

'M. E. Bussell and G. A. Somorjai, J. Catal. 106, 93 (1987).

${ }^{2}$ M. E. Bussell, A. J. Gellman, and G. A. Somorjai, Catal. Lett. 1, 195 (1988).

${ }^{3}$ M. Salmeron, G. A. Somorjai, and R. R. Chianelli, Surf. Sci. 127, 526 (1983).

${ }^{4}$ V. Maurice, L. Peralta, Y. Berthier, and J. Oudar, Surf. Sci. 148, 623 (1984).

${ }^{5}$ L. J. Clarke, Surf. Sci. 102, 331 (1981).

${ }^{6}$ F. Zaera, E. B. Kollin, and J. L. Gland, Surf. Sci. Lett. 166, L149 (1986).

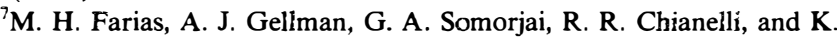
S. Liang, Surf. Sci. 140, 181 (1984).

${ }^{8}$ C. C. Knight and G. A. Somorjai, Surf. Sci. 240, 101 (1990).

${ }^{9}$ C. C. Knight and G. A. Somorjai, Surf. Sci. 272, 326 (1992).

${ }^{10}$ J. C. Dunphy, C. Kright, P. Sautet, D. F. Ogletree, G. A. Somorjai, and M. B. Salmeron, Surf. Sci. (in press).

"D. G. Kelley, R. F. Lin, M. A. Van Hove, and G. A. Somorjai, Surf. Sci. 224, 97 (1989).

${ }^{12}$ P. J. Rous, D. Jentz, D. G. Kelley, R. Q. Hwang, M. A. Van Hove, and G. A. Somorjai, The Structure of Surfaces III, Proceedings of the 3rd International Conference on the Structure of Surfaces (ICSOS-III), edited by S. Y. Tong, M. A. Van Hove, K. Takayanagi, and X. D. Xie (Springer, Berlin, 1991), p. 432.

${ }^{13}$ D. Jentz and M. A. Van Hove (private communication).

${ }^{14}$ B. Marchon, P. Bernhardt, M. E. Bussell, G. A. Somorjai, M. Salmeron, and W. Siekhaus, Phys. Rev. Lett. 60, 1166 (1988).

${ }^{15} \mathrm{X}$. W. Wang and S. G. Louie, Surf. Sci. 226, 25 ? (1990).

${ }^{16}$ D. M. Zeglinski, D. F. Ogletree, T. P. Bcebe, Jr., R. Q. Hwang, G. A Somorjai, and M. Salmeron, Rev. Sci. Instrum. 61, 3769 (1990). 
${ }^{17}$ C. Wagner, J. Chem. Phys. 21, 1819 (1953).

${ }^{18}$ A. J. Gellman, J. C. Dunphy, and M. Salmeron, Langmuir 8, 534 (1992).

${ }^{19}$ V. Maurice and P. Marcus, Surf. Sci. 262, L59 (1992).

20J. C. Dunphy, D. F. Ogletree, M. Salmeron, P. Sautet, and M.-L. Bocquet, Uitramicros. 42-44, 490 (1992).
${ }^{2 i}$ S. Rousset, S. Gauthier, O. Sibouiet, W. Sachs, M. Belin, and J. Klein, J. Vac. Sci. Technol. A 8, 302 (1990).

${ }^{22}$ J. M. MacLauren, J. B. Pendry, P. J. Rous, D. K. Saldin, G. A. Somorjai, M. A. Van Hove, and D. D. Vvedensky, A Handbook of Surface Siructures (Reidel, Dordrecht, 1987). 